\title{
Diseño de sistema de monitoreo remoto para evaluación de la corrosión en estructuras de concreto reforzado sometidas a ion cloruro
}

\section{Design of a Remote Monitoring System for Evaluation of Corrosion in Reinforced Concrete Structures under Chloride Ion Attack}

\author{
Roa-Rodríguez Guillermo \\ Universidad Militar Nueva Granada, Colombia \\ Correo:guillermoroa.rrg@gmail.com \\ Aperador-Chaparro William \\ Universidad Militar Nueva Granada, Colombia \\ Correo:g.ing.materiales@gmail.com
}

\author{
Delgado-Tobón Emilio \\ Universidad Militar Nueva Granada, Colombia \\ Correo:Arnoldo.umng@unimilitar.edu.co
}

Información del artículo: recibido: agosto de 2014, reevaluado: octubre de 2014, aceptado: enero de 2015

\begin{abstract}
Resumen
En el presente trabajo se diseñó y construyó un equipo de monitoreo remoto que permite obtener los potenciales de corrosión en los aceros de refuerzo inmersos en concreto reforzado, los cuales se sometieron previamente al ataque de cloruros en un ambiente hostil. El sistema de monitoreo, basado en la norma ASTM C876-91, permite determinar desde 0 a 100\% la probabilidad de corrosión sobre las muestras evaluadas. El sistema otorga la facilidad para realizar su instalación en campo, siempre y cuando exista cobertura de red celular, puede operarse de forma remota mediante mensajes de texto para iniciar o detener las mediciones, cuyos resultados son almacenados en un datalogger local en tarjetas microSD, posteriormente se envían mediante el servicio general de paquetes vía radio (GPRS) a un servidor Web, que permite dar accesibilidad a los datos mediante una página Web, en donde se puede observar el resultado del ensayo de forma gráfica. Las muestras de hormigón usadas como referencia para el monitoreo de la degradación fueron inmersas en ion cloruro $(3.5 \% \mathrm{NaCl})$ durante 12 meses. Los datos de potencial de corrosión generaron la respuesta de la interfase medio de exposición-hormigón, que corresponde a un sistema con $90 \%$ de probabilidad de corrosión.
\end{abstract}

\section{Descriptores:}

- corrosión

- monitoreo remoto

- refuerzos de acero

- diseño de equipo

- estructuras de concreto

- ataque de cloruros 


\begin{abstract}
In this paper it was designed and built a remote monitoring equipment that allows to obtain the corrosion potential in reinforcing steels embedded in reinforced concrete, which were previously subjected to chloride attack in a hostile environment. The monitoring system, based on ASTM standard C876-91, determines from $0 \%$ to $100 \%$ the probability of corrosion on the samples tested. The system provides ease of perform field installation, if there is cellular network coverage, and may be operated remotely using text messages to start and stop measurements, whose results are stored in a local data logger on microSD cards and then are sent via the general packet radio service (GPRS) to a web server which allows to access to the data via a web page, where the test results can be seen graphically. The concrete samples used as reference for monitoring degradation were immersed in chloride ion $(3.5 \% \mathrm{NaCl})$ for 12 months. Data for corrosion potential were generated through the exposureconcrete interface, corresponding to a system with a $90 \%$ probability of corrosion.
\end{abstract}
Keywords:
- corrosion
- in situ monitoring
- rebars
- equipment design
- concrete structures
- chloride attack

\section{Introducción}

La corrosión de los refuerzos de acero es común en estructuras de concreto reforzado alrededor del mundo. Este fenómeno causa un deterioro prematuro de infraestructuras civiles tales como, edificaciones, vías, puentes, plataformas marinas, tuberías y represas (Tang et al., 2012). En la mayoría de las estructuras, los refuerzos proveen la seguridad estática de la construcción, y teniendo en cuenta el papel que desempeñan en la capacidad de soportar y transportar cargas, la medición y la información acerca del estado de corrosión actual del material metálico es por demás prioritario (Mietz et al.,1996). Las estructuras de concreto reforzado proporcionan una excelente vida de servicio bajo condiciones ambientales específicas; en concretos provenientes de buenos procesos de fabricación y de buena calidad, el riesgo de corrosión es mínimo y normalmente estos proveen una aceptable protección química y física para los aceros embebidos (Pradhan et al., 2009; Melchers et al., 2006; Almusallam et al., 2011). La corrosión de los refuerzos del concreto se considera generalmente como un proceso electroquímico que deteriora la capa protectora de los refuerzos de acero (película protectora de óxido que se forma previamente debido al alto nivel de alcalinidad del concreto); habitulamente dicho daño se genera por la presencia de iones de cloruro al nivel de los refuerzos, los cuales pueden originarse por el uso de contaminantes en la mezcla o por el ambiente circundante en el estado sólido del material (Pradhan et al., 2009; Roa et al., 2013a). Dichos iones de cloruros se pueden presentar en el concreto de tres maneras: enlazados, adsorbidos y disueltos en el agua que se conserva en los poros, lo que constituye la disolución poro. Los iones cloruro que son dañinos para el acero de refuerzo son los disueltos o libres, pero debido a los equilibrios que se presentan es posible que los adsorbidos se incorporen a la disolución y se tornen peligrosos (Roa et al., 2013; Aperador et al., 2012; Fajardo et al., 2010; Montoya et al., 2009).

La difusión es el mecanismo capaz de llevar los iones cloruro al nivel de los refuerzos de hierro, por lo tanto, es capaz de acelerar el proceso corrosivo; la difusión ocurre una vez que el concreto saturado se expone a la solución de cloruros a presión normal, un gradiente de concentración de cloruros se crea entre la superficie y el medio poroso interior, dicho gradiente se constituye como la fuerza que impulsa el proceso corrosivo (Ahmed et al., 2013). La corrosión de las estructuras de concreto se describe como un proceso en dos etapas: la etapa de iniciación y la etapa de propagación (Ahmed et al., 2013; Tuuti, 1982; Ahmed et al., 2014); específicamente para el fenómeno corrosivo causado por cloruros, la etapa de iniciación corresponde al periodo durante el cual los cloruros penetran el concreto, pero ningún deterioro se observa. El periodo de iniciación se define por el tiempo en el que la concentración de cloruros en la superficie del acero alcanza el umbral crítico. Por su parte, la etapa de propagación corresponde al periodo en donde los productos de corrosión se acumulan e inician la fractura y finalmente la falla del concreto (Ahmed et al., 2014). Estos iones agresivos penetran el concreto a través de las micro-grietas y los espacios generados por los poros en la matriz de cemento; la tasa de penetración depende primordialmente de la calidad del concreto, particularmente, de la relación agua/cemento de la mezcla y de la presencia de cementantes suplementarios (humo de sílice, ceniza volante o escoria), además de los sistemas protectores que 
retrasan el ingreso de cloruros (Ahmed et al., 2014; Kropp et al., 1995).

Debido a que los productos generados por el fenómeno de corrosión aumentan para posteriormente conviertirse en grietas sobre el concreto, es necesario un monitoreo oportuno antes de que se evidencie un deterioro crítico. Para este fin, los métodos de evaluación no destructivos se han implementado recientemente para estimar el nivel de corrosión en las estructuras. Hasta el momento, las dos técnicas comercialmente viables se basan en el potencial de circuito abierto y en la resistencia a la polarización lineal. La medición de potenciales de circuito abierto es práctica y ampliamente usada para identificar la presencia de fenómenos corrosivos (Yokota et al., 2004). Los refuerzos embebidos en concreto, tanto activos como pasivos, presentan una diferencia de potencial electroquímico hasta de $500 \mathrm{mV}$, que provoca un flujo de corriente; dicho campo eléctrico, junto con la corriente de corrosión entre las regiones activas y pasivas de los refuerzos se puede medir experimentalmente con un electrodo de referencia apropiado (denominado media celda) en la superficie del concreto. Los resultados de estas medidas pueden representarse como líneas equipotenciales que permiten localizar los refuerzos corroídos en los valores de potenciales más negativos (Roa et al., 2013b; Elsener et al., 2001). La técnica de medición se encuentra estandarizada bajo la norma ASTM C876-91, aplicable para la evaluación en sitio e independiente del tamaño del refuerzo y de la profundidad dentro de la pasta de concreto. La técnica de medición permite, mediante el uso de un voltímetro, obtener un delta de voltaje entre un refuerzo de la estructura y un electrodo de referencia. El electrodo de referencia consiste en un contenedor compuesto por un material dieléctrico no reactivo con cobre o con sulfato de cobre, un material poroso que permanece húmedo y un alambre de cobre, el cual se intruduce en el contenedor con una solución saturada de sulfato de cobre (ASTM Standard C876-09, 2003).

El control de calidad, mantenimiento y planeación de restauración de las estructuras bajo ataques corrosivos, requieren monitoreo e inspecciones no destructivas que permitan detectar la corrosión en etapas tempranas. Las pérdidas por corrosión consumen gran parte del presupuesto del país debido a las restauraciones o reconstrucciones necesarias luego del deterioro. Un monitoreo apropiado de las estructuras en riesgo de sufrir este fenómeno y la toma de medidas adecuadas a tiempo pueden acarrear grandes ahorros, además, las operaciones de reparación en sí mismas son complejas y requieren un tratamiento especial en las zonas con fractura, en su mayoría, la ex- pectativa de vida de reparación es limitada. Por lo tanto, el monitoreo de corrosión puede proporcionar información mucho más completa, capaz de indicar la condición de cambio de la estructura de acuerdo al paso del tiempo y a la exposición del ambiente corrosivo (Saraswathy et al., 2007). El estado del arte previo ha mostrado que los diseños e implementaciones actuales tienen una gran limitante en su flexibilidad, pues dependen en todo momento de un hardware; específicamente un computador para procesamiento que esté vinculado directamente a los sensores de medida, encargado de almacenar y procesar las mediciones y presentar los reportes requeridos. A su vez, dicho sistema debe operarse en el sitio donde se lleva a cabo el estudio por un usuario, lo que aumenta costos por el desplazamiento de equipo y personal (Poursaee, 2009; Thirumalai et al., 2006; Kumar et al., 2006; Duffó et al., 2007; Hudson et al., 2002; Rengaswamy et al., 2004; Minrago et al., 1996).

Este trabajo presenta la evaluación de la corrosión en estructuras de concreto reforzado sometidas al ataque de cloruros mediante el desarrollo de un sistema de monitoreo in situ para estructuras de concreto reforzado, basado en la norma ASTM C876-91, este evita el desplazamiento a campo tanto de equipo como de operarios y dispone consulta inmediata de la información proveniente de las mediciones adquiridas, con las cuales es posible determinar la probabilidad del estado corrosivo de los aceros de refuerzo embebidos.

\section{Materiales y métodos}

La técnica electroquímica para la evaluación del grado de deterioro del acero de refuerzo embebido en concreto, de forma no destructiva y más utilizada, es la medición de potencial de media celda (Ecorr) denominada a partir de este punto como potencial de corrosión, en donde la aplicación de pequeñas perturbaciones en la interfase, que mantienen un estado termodinámimente estable o en equilibrio, aportan información acerca de las condiciones de la interfase acerohormigón y con ello, del grado de deterioro que se presente en la armadura.

Diseño del equipo de monitoreo de corrosión remoto

Se diseñó un sistema de monitoreo remoto (in situ) basado en el estándar ASTM C876-09; el diseño permite adaptar la técnica de medida de potenciales de media celda en una variación in situ, con la cual es posible evitar el desplazamiento de personal y equipo de medida 
cada vez que se requiera conocer el estado corrosivo de la estructura en algún lugar, esto conlleva a una reducción en el costo para un proyecto de protección de los refuerzos embebidos.

Las mediciones electroquímicas de potencial de media celda forman parte de las técnicas parcialmente no destructivas (ya que hay que afectar la estructura en un punto específico), de costo aceptable, que permite evaluar la probabilidad de corrosión de los aceros que forman parte de la estructura de concreto. La técnica de medición se encuentra estandarizada bajo la norma ASTM C876-91, aplicable para la evaluación en sitio, e independiente del tamaño del refuerzo y de la profundidad dentro de la pasta de concreto. La técnica de medición permite, mediante el uso de un voltímetro, obtener un delta de voltaje entre un refuerzo de la estructura y un electrodo de referencia (figura 1). El electrodo de referencia consiste en un contenedor compuesto por un material dieléctrico no reactivo con cobre o con sulfato de cobre, un material poroso que permanece húmedo, así como un alambre de cobre, que es inmerso en el contenedor con una solución saturada de sulfato de cobre. Luego se dispone el electrodo de referencia sobre la superficie del concreto y se realiza su conexión al terminal negativo (tierra) del voltímetro, posteriormente se realiza un contacto eléctrico directo al acero de refuerzo. Esta disposición de media celda arroja un diferencial de voltaje correspondiente a la probabilidad de corrosión (tabla 1).

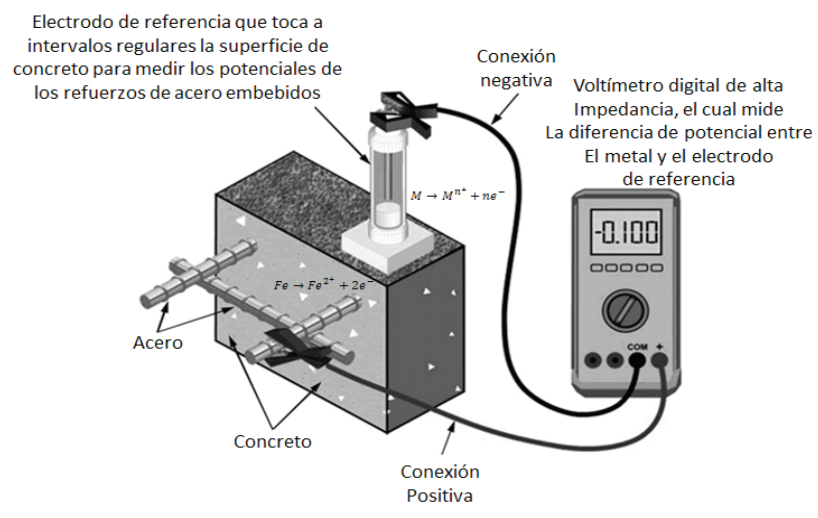

Figura 1. Técnica de medición tomada de la norma ASTM C876-91 (ASTM Standard C876-09, 2003)

Tabla 1. Potencial de corrosión según norma ASTM C876 (1991)

\begin{tabular}{cc}
\hline $\begin{array}{r}\text { Voltaje medido vs } \\
\text { Electrodo de Cobre/ } \\
\text { Sulfato de Cobre }(\mathrm{V})\end{array}$ & \multicolumn{1}{c}{ Probabilidad de corrosión } \\
\hline$>-0.2 \mathrm{~V}$ & $10 \%$ o menos \\
$-0.2 \mathrm{~V} \mathrm{a}-0.35$ & región de incertidumbre \\
$<-0.35$ & $90 \%$ o más \\
\hline
\end{tabular}

Con base en la norma, se realizó el diseño del sistema en su variación embebida, con el fin de obtener una media celda de forma indefinida al interior del concreto, que permite obtener el potencial de corrosión correspondiente y asociarlo con el estado corrosivo. La adaptación embebible se realizó a partir de un electrodo de referencia embebible de Cobre/Sulfato de Cobre ( $\mathrm{Cu} /$ $\mathrm{CuSO}_{4}$ ) y una barra de refuerzo del mismo material de la estructura, los cuales finalmente se recubrieron por el material nativo de la estructura, en este caso, mortero. La conexión de los alambres provenientes del electrodo de referencia y del segmento de acero estructural se llevó a un conector XLR de 3 pines, que fue lo suficientemente robusto para realizar el aislamiento de la señal de voltaje del ruido externo, lo que permite estabilizar su lectura. Antes de la instalación, el electrodo de referencia fue pre-humedecido en un recipiente con agua potable durante 30 segundos, luego se insertó en la sonda y el agua restante fue vertida en el agujero (de un diámetro de 3 pulgadas y una profundidad de 10 pulgadas). Este procedimiento se realizó porque crea la condición para que el material que rodea al electrodo y el mortero penetren y se fijen a los poros del electrodo de referencia (figura 2).

El potencial de media celda que proporciona la sonda embebible posteriormente se lleva al sistema de adquisición de datos (figura 3), este permite realizar la adquisición de dicha variable mediante canales análogos, configurados en un rango de $\pm 1200 \mathrm{mV}$, a intervalos de 1 segundo (obtener mayores tasas de adquisición resulta innecesario, pues la variación del voltaje en ensayos de corta duración es despreciable). Asimismo, el sistema hace uso de un módulo de tarjetas microSD, que permite crear un archivo en formato de texto plano en donde se almacenan los datos medidos en el ensayo. El proceso de adquisición toma 15 minutos, tiempo suficiente para tomar 900 lecturas desde la media celda instalada.

Se adicionó posteriormente un módulo GSM/GPRS M95 (Global System for Mobile communications/General Packet Radio Service) que permite usar la red celular insertando una SIM CARD de cualquier operador de telefonía móvil, para que le asigne al equipo un único número celular capaz de recibir y enviar mensajes de texto, herramienta con la que se realiza el comando del equipo de forma remota. Con esta tarjeta, es posible entablar la comunicación con el equipo a través de mensajes, los cuales indican el inicio de los ensayos y la toma de los voltajes de la media celda embebida. Una vez que las mediciones se han almacenado en la memoria física, el módulo de comunicación habilita la función FTP (File Transfer Protocol) con que se realiza el 


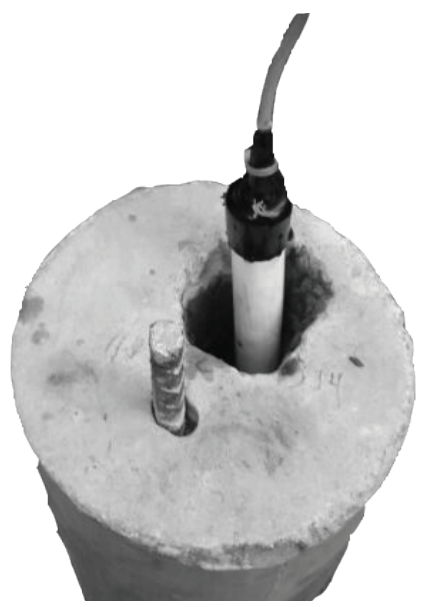

a) Inserción del electrodo de referencia

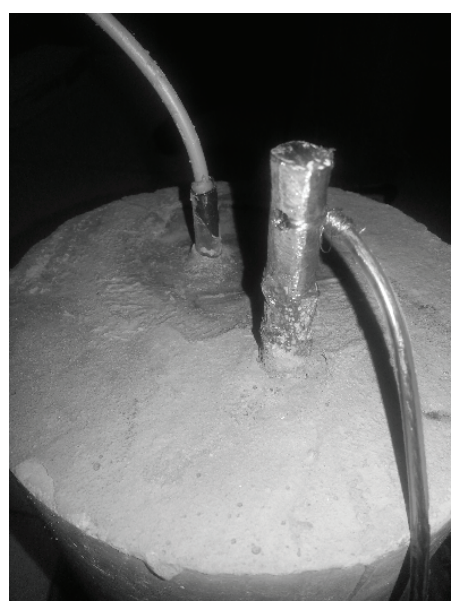

b) Conexión eléctrica del refuerzo

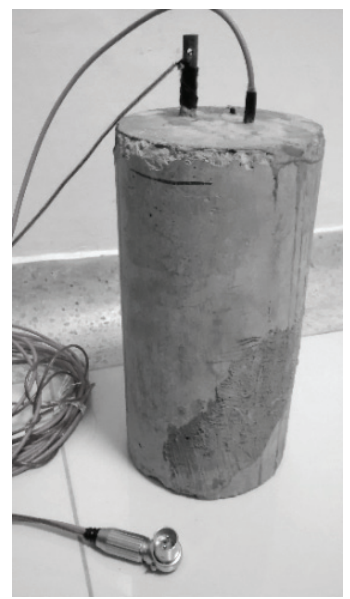

c) Contacto eléctrico con el conector XLR

Figura 2. Implementacón de la sonda embebible

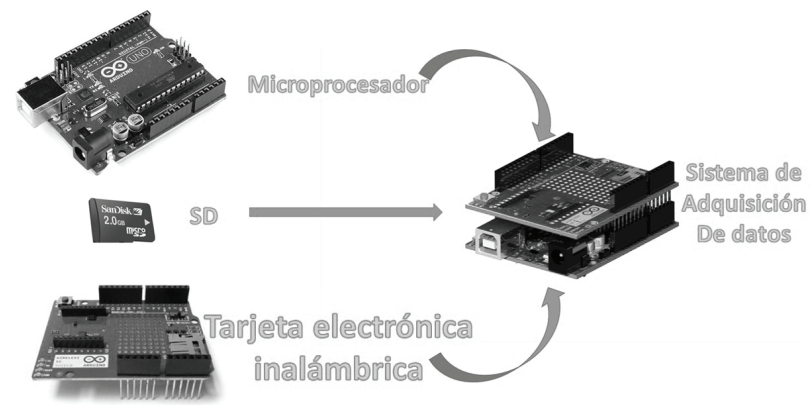

Figura 3. Sistema de adquisición de datos

envío del archivo de texto plano a un servidor Web, el cual alberga un supervisorio y una base de datos para realizar la gestión de los datos y arrojar el reporte al usuario. El supervisorio Web se alberga en un servidor Web dedicado, el cual gestiona una base de datos encargada de recibir y ordenar los valores de voltaje enviados desde el equipo; así mismo el servidor se configura para realizar el tratamiento de los datos almacenados en la base de datos, con el fin de darles un formato gráfico y disponerlos en una página Web accesible mediante un link. La página muestra la representación gráfica de la tendencia de datos (figura 4), mediante la segmentación de la zona de trazo en 3 regiones de distribución probabilística acorde a la norma soporte del presente trabajo (según la tabla 1 presentada anteriormente).

Vida útil del sistema y estrategias de mantenimiento La vida útil del sistema se cumple por la longevidad y desempeño del electrodo embebible usado, la cual va- ría según el fabricante. Como referencia, se tomó la vida útil del electrodo empleado en el diseño, el cual corresponde a 30 años. Durante dicho periodo los potenciales de corrosión entregados por el electrodo de referencia se mantienen estables e independientes de factores como humedad y temperatura.

Como estrategias de mantenimiento es necesario realizar una visita a campo cada vez que la memoria microSD alcance su capacidad máxima de almacenamiento (la cual puede ser de cualquier tamaño) con el fin de liberar capacidad de procesamiento para poder albergar ensayos venideros.

\section{Resultados y discusiones}

Ensayo de monitoreo de corrosión debido a ataque de cloruros sobre probetas

Se fabricaron probetas con un diámetro de $150 \mathrm{~mm}$ y $300 \mathrm{~mm}$ de longitud con refuerzos de acero de $1 \frac{1}{2}$, las

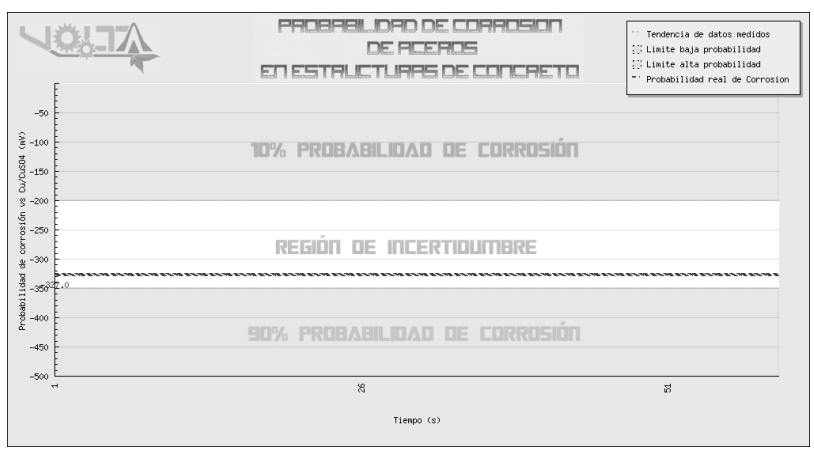

Figura 4. Supervisorio Web 
cuales se construyeron usando cemento Portland tipo I. Posteriormente se sometieron a inmersión en una solución que simula las condiciones marinas $(\mathrm{NaCl}$ $3.5 \%)$. El equipo se dispuso para realizar el monitoreo de probetas sometidas a ataque de cloruros, almacenando los valores de potencial de circuito abierto en intervalos periódicos, durante un lapso total de 12 meses, iniciando el ataque de cloruros transcurridas 140 días. La caracterización electroquímica de contraste se realizó en un potenciostato/galvanostato Gamry modelo PCI 4 mediante las técnicas de potencial de circuito abierto, empleando la misma celda utilizada para el monitoreo in situ.

El comportamiento del potencial de media celda durante el ensayo, permitió determinar una probabilidad de corrosión de $10 \%$ durante el tiempo que transcurrió la prueba sin realizar la inmersión en el medio saturado de iones cloruro (potenciales menores a $-147 m V$ y mayores a $-128 m V$ ). Una vez se incluyó el medio agresivo en el procedimiento, los potenciales aumentaron en la región negativa de forma progresiva hasta alcanzar una probabilidad de corrosión de $90 \%$ (potenciales mayores a $-350 \mathrm{mV}$ ) (figura 5). Luego de finalizar la adquisición de las mediciones provenientes del equipo desarrollado y del equipo patrón, se realizó la comparación, evidenciando un error mínimo de $0.029 \%$ y un error máximo de $2.2044 \%$. Asimismo se encontró un error medio de $0.93 \%$ sobre las 49 mediciones realizadas durante los 12 meses.En la figura 6 se observa la micrografía de la superficie de acero embebido en el concreto portland ordinario, el cual ha estado expuesto a ion cloruro. Se realizó solamente sobre esta

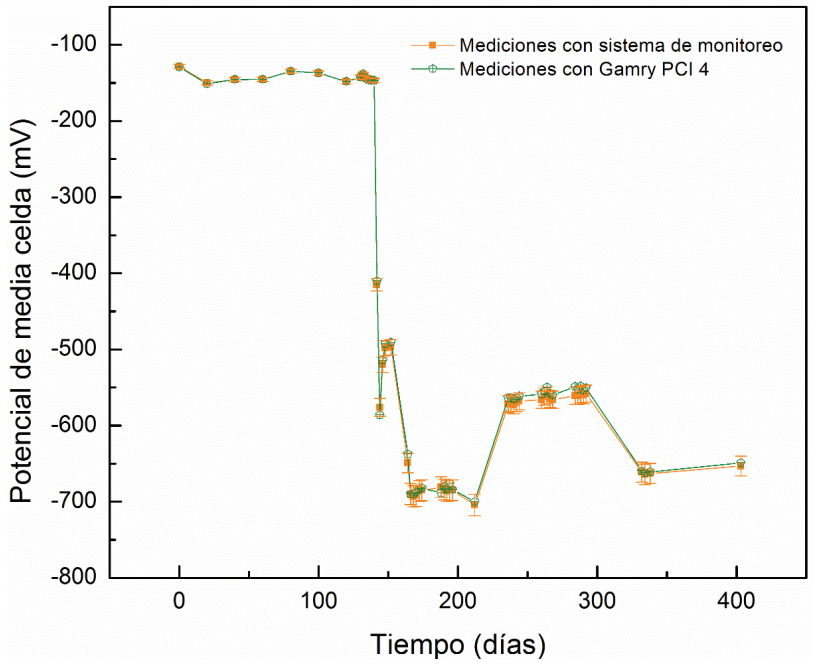

Figura 5. Comparación entre las medidas tomadas durante 12 meses por el sistema de monitoreo desarrollado y el sistema de comparación Gamry PCl 4

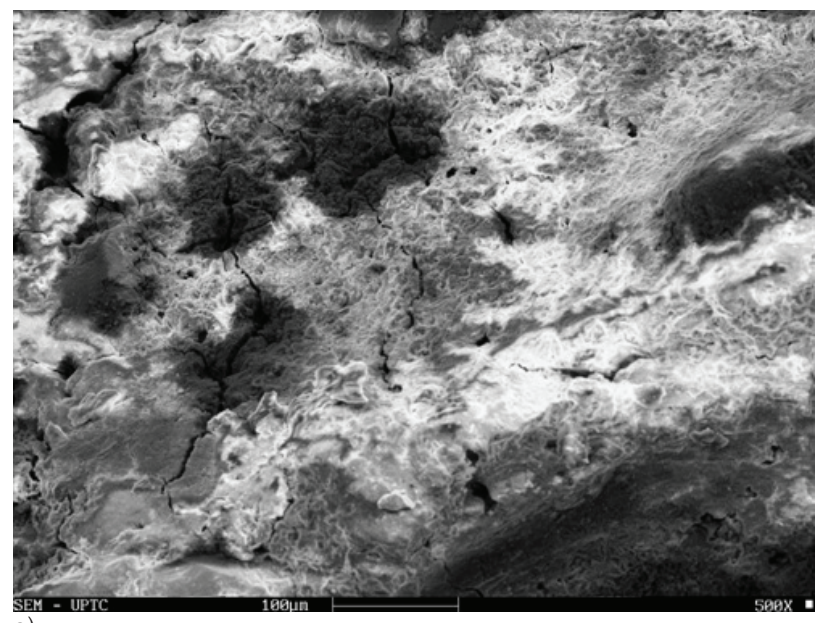

a)

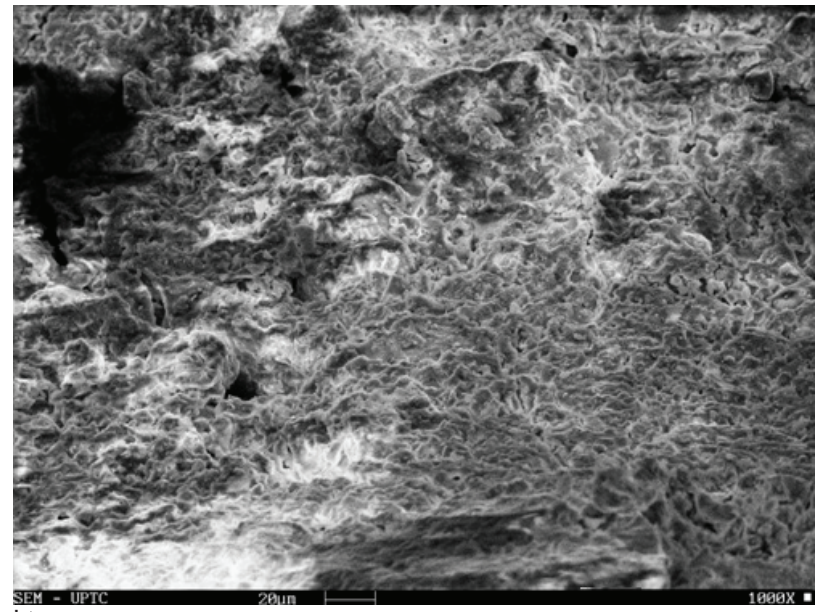

b)

Figura 6. Observación microscópica, a) 500X de magnificación del acero en contacto con el concreto OPC, b) 1000X de magnificación de los productos de corrosión producidos por los procesos de degradación por el contenido del ion cloruro

superficie debido a que la observación con menores magnificaciones revelaron un estudio similar para probetas expuestas a ambiente natural. En la figura 6a, se localiza una área en donde se encuentra la mayor concentración de grietas. Por medio del análisis con EDS, se evidencia que las zonas oscuras corresponden a la magnetita y se deduce que estas zonas oscuras son las que generan una zona de mayor concentración de grietas por la dilatación de la magnetita, en esta micrografía se puede aseverar que las primeras capas formadas sobre el acero corresponden a la magnetita y que la siguiente capa se debe a otros productos de corrosión. En la magnificación a 1000X (figura 6b) realizada sobre la superficie del acero embebido en Portland se observan los productos de corrosión de la superficie externa, es- 


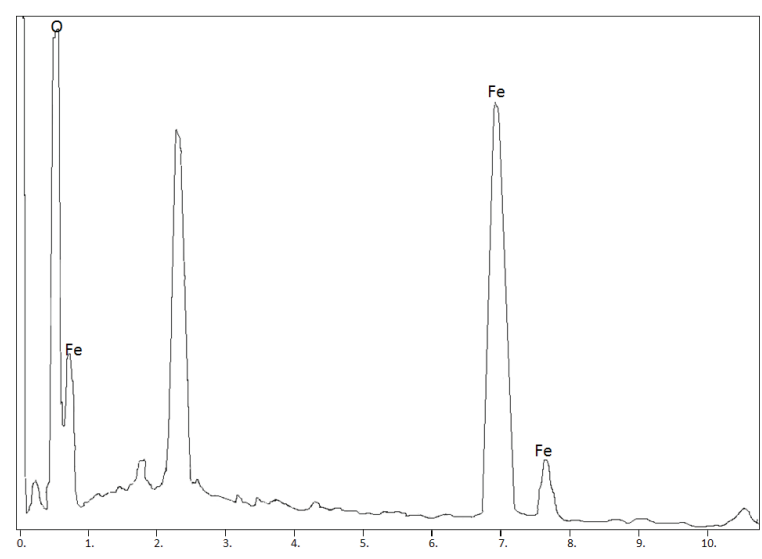

Figura 7. a) ensayos EDX sobre muestras corroídas y b) presencia de Magnetita

tos productos según EDX corresponden a goethita y lepidocrocite.

La técnica EDX es un ensayo semi-cuantitativo, por lo que es útil para proporcionar información sobre la presencia o ausencia de elementos que componen un material, los datos cuantitativos que se producen solo pueden tratarse como una estimación de la composición química. La figura 7 muestra el resultado del ensayo realizado, evidenciando magnetita compuesta por Hierro $(\mathrm{Fe})$ y picos de Oxígeno $(\mathrm{O})$ (Vera et al., 2009; Singh et al., 2012).

\section{Conclusiones}

A través de este estudio se desarrolló un equipo de monitoreo remoto de corrosión capaz de adquirir potenciales de media celda, para determinar a partir de estos, las probabilidades de corrosión (10 a 90\%) con errores medios de $0.029 \%$ con base en equipos comerciales patrón (Gamry PCI 4). El equipo desarrollado se expuso a ambientes corrosivos saturados, como el ambiente marino (saturación de 3.5\% de ion cloruro), en donde se encontró que es posible monitorear los potenciales de media celda y su evolución en el tiempo de forma remota y estable, sin superar errores máximos de $2.2044 \%$.

El estudio permitió obtener un sistema capaz de realizar mediciones de potencial de media celda con una precisión de $\pm 1 \mathrm{mV}$, cuya tasa de muestreo es de 1 segundo, garantizando la obtención de un potencial de media celda verídico. El sistema desarrollado posee una gran versatilidad en cuanto a su lugar de ubicación, ya que el uso de la red celular que dispone (GSM), le habilita para comandar de forma remota independiente de la distancia desde donde se encuentre el centro de control. Asimismo, proporciona ventajas sobre el personal y equipo necesario, pues reduce costos al disponer directamente la información en un servidor Web accesible por cualquier usuario con una conexión mínima a internet.

El monitoreo corresponde con el estado corrosivo de los refuerzos de acero, evidenciado mediante microscopía electrónica de barrido, al mismo tiempo es remarcable la presencia de productos de corrosión en forma de magnetita, goethita y lepidocrocite hallados mediantes EDX

\section{Agradecimientos}

Producto derivado del proyecto ING1527 financiado por la Vicerrectoría de Investigaciones de la Universidad Militar Nueva Granada-Vigencia 2014.

\section{Referencias}

Ahmed H.I., Hodhod O.A. Developing an artificial neural network model to evaluate chloride diffusivity in high performance concrete. HBRC journal, volumen 9, abril de 2013: 15-21.

Ahmed H.I., Hodhod O.A. Modeling the service life of slag concrete exposed to chlorides. Ain Shams Engineering Journal, volumen 5, marzo de 2014: 49-54.

Almusallam A.A. Effect of degree of corrosión on the properties of reinforcing Steel bars. Construction and Building Materials 2001, volumen 15, diciembre de 2011: 361-368.

Aperador W., Vera R., Carvajal A.M. Industrial byproduct-based concrete subjected to carbonation. Electrochemical behavior of steel reinforcement. International Journal of Electrochemical Science, volumen 7, diciembre de 2012: 12870-12882.

ASTM Standard C876-09, 1991. Standard test method for corrosion potentials of uncoated reinforcing steel in concrete, ASTM International, West Conshohocken, PA, 2003, DOI: 10.1520/C0876-09.

Duffó G., Farina S.B., Arva E.A., Giordano C.M., Lafont C.J. Sistema integrado de monitoreo de la corrosión del acero en el hormigón armado, Congreso SAM/CONAMET 2007, septiembre 2007.

Elsener B. Half-Cell potential mapping to asses repair work on RC structures. Construction and Building Materials, volumen 15, abril de 2001: 133-139.

Fajardo S., Bastidas D.M., Ryan M.P., Criado M., McPhail D.S., Bastidas J.M. Low-nickel stainless steel passive film in simulated concrete pore solution: A SIMS study. Applied Surface Science, volumen 256, agosto de 2010: 6139-6143.

Hudson J.K., Jones S.H, Kelly R.G, Ross R.A Embeddable corrosion monitoring-instrument for steel reinforced structures, solicitud de patente, enero de 2002, documento WO0206764.

Kropp J., Hilsdorf H.K. Performance criteria for concrete durability, RILEM Report 12, E \& FN Spon, Londres, 1995.

Kumar K., Muralidharan S., Manjula T. Sensor systems for corrosion monitoring in concrete structures. Sensors \& Transducers, volumen 67, mayo de 2006: 553-560. 
Melchers R.E., Li C.Q. Phenomenological modeling 7of reinforcement of reinforcement corrosión in marine enviroments. Aci Materials Journal, volumen 1, enero de 2006: 103:25-32.

Mietz J., Isecke B. Monitoring of concrete structures with respect to rebar corrosion. Construction and Building Materials, volumen 10, julio de 1996: 367-373.

Minrrago M.F. Degradación y conservación del patrimonio arquitectónico, Madrid, editorial Complutense, 1996. 504 p.

Montoya R., Aperador W., Bastidas D.M. Influence of conductivity on cathodic protection of reinforced alkali-activated slag mortar using the finite element method. Corrosion Science, volumen 51, diciembre de 2009: 2857-2862.

Pradhan B., Bhattacharjee B. Performance evaluation of rebar in chloride contaminated concrete by corrosion rate. Construction and Building Materials, volumen 23, junio de 2009: 2346-2356.

Poursaee A. Automatic system for monitoring corrosion of steel in concrete. Advances in Engineering Software, volumen 40, noviembre de 2009: 1179-1182.

Rengaswamy S., Hassan M., Terry E. Embeddable corrosion rate meters for remotely monitoring structures, solicitud de patente, enero de 2004, documento WO2004010104.

Roa-Rodríguez G., Aperador W., Delgado A. Calculation of chloride penetration profile in Concrete Structures. International Journal of Electrochemical Science, volumen 8, abril de 2013a: 5022-5035.

Roa-Rodríguez G., Aperador W., Delgado E. Resistance to Chlorides of the Alkali-Activated Slag Concrete. International Journal of Electrochemical Science, volumen 9, noviembre de 2013b: 282-291.

Saraswathy V., Song H. Corrosion monitoring of reinforced concrete structures-a review. International Journal of Electrochemical Science, volumen 2, enero de 2007: 1-28.

Singh J.K., Singh D.D.N. The nature of rusts and corrosion characteristics of low alloy and plain carbon steels in three kinds of concrete pore solution with salinity and different $\mathrm{pH}$. Corrosion Science, volumen 56, marzo 2012: 129-142.
Tang F., Chen G., Brow R., Volz J., Koenigstein M. Corrosion resistance and mechanism of steel rebar coated with three types of enamel. Corrosion. Science, volumen 59, junio de 2012: 57-168.

Thirumalai-Parthiban R., Ravi G.T.P. Potential monitoring system for corrosion of steel in concrete. Advances in Engineering Software, volumen 37, junio de 2006: 375-381.

Tuutti K. Corrosion of steel in concrete. Swedish Cement and Concrete Research Institute, octubre de 1982, Núm. 4, pp. 468.

Vera R., Villarroel M., Carvajal A.M., Vera E., Ortiz C. Corrosion products of reinforcement in concrete in marine and industrial environments. Materials Chemistry and Physics, volumen 114, marzo de 2009: 467-474.

Yokota M., Ohtsu M., Kyung J., Leelalerkiet V. Analysis of halfcell potential measurement for corrosion of reinforced concrete. Construction and Building Materials, volumen 18, abril de 2004: 155-162.

\section{Este artículo se cita:}

\section{Citación estilo Chicago}

Roa-Rodríguez, Guillermo, William Aperador-Chaparro, Emilio Delgado-Tobón. Diseño de sistema de monitoreo remoto para evaluación de la corrosión en estructuras de concreto reforzado sometidas a ion cloruro. Ingeniería Investigación y Tecnología, XVI, 04 (2015): 565-572.

\section{Citación estilo ISO 690}

Roa-Rodríguez G., Aperador-Chaparro W., Delgado-Tobón E. Diseño de sistema de monitoreo remoto para evaluación de la corrosión en estructuras de concreto reforzado sometidas a ion cloruro. Ingeniería Investigación y Tecnología, volumen XVI (número 4), octubre-diciembre 2015: 565-572.

\section{Semblanzas de los autores}

Roa-Rodríguez Gillermo. Ingeniero en mecatrónica por la Universidad Militar Nueva Granada (julio de 2011). Especialista en gerencia integral de proyectos por la Universidad Militar Nueva Granada (noviembre de 2013). Candidato a magister en maestría en ingeniería mecatrónica de la Universidad Militar Nueva Granada. Es asistente de investigación en el grupo de investigación VOLTA, cuya calificación A1 lo ubica en el más alto estándar en grupos de investigación colombianos.

Aperador-Chaparro William. Profesor investigador de la Universidad Militar Nueva Granada, doctor en ingeniería por la Universidad del Valle. Obtuvo el título de magister en metalurgia y ciencia de los materiales por la Universidad Pedagógica y Tecnológica de Colombia (UPTC-Tunja). Cuenta además con el título de físico en la misma Universidad. Realizó una pasantía internacional en el centro nacional de investigaciones metalúrgicas de España, en el laboratorio de ingeniería de materiales y durabilidad. Fue profesor en la escuela de física de la UPTC-Tunja, Escuela de ingeniería de materiales de la Universidad del Valle y Escuela de Ingeniería Mecánica de la Escuela Colombiana de Ingeniería.

Delgado-Tobón Emilio. Ingeniero mecánico de la Universidad Nacional y Magíster en ingeniería mecánica por la Universidad de los Andes. Profesor de planta en la Universidad Militar Nueva Granada y en la Escuela Colombiana de Ingeniería. Cuenta con experiencia en diseño, montaje y puesta en marcha de líneas de producción de materiales poliméricos en la industria. Es experto en mezclas de polímeros y ha participado en el desarrollo de proyectos ambientales, ejecución de planes de mantenimiento preventivo y correctivo en equipos industriales, además de desempeñarse como jefe de producción y control de calidad de equipos. 